\title{
Type I Diabetic Akita Mouse Model is Characterized by Abnormal Cardiac Deformation During Early Stages of Diabetic Cardiomyopathy with Speckle- Tracking Based Strain Imaging
}

\author{
Yingchao Zhou ${ }^{\mathrm{a}}$ Hong Xiao $^{\mathrm{b}}$ Jianfei Wu $\mathrm{u}^{\mathrm{a}}$ Lingfeng Zhac Mengchen Zhou

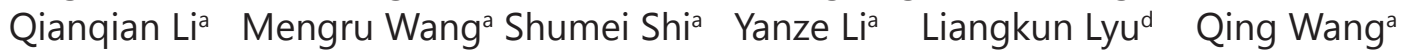 \\ Xin Tu ${ }^{a}$ Qiulun $\mathrm{Lu}^{\mathrm{a}}$
}

aKey Laboratory of Molecular Biophysics of the Ministry of Education, College of Life Science and Technology and Center for Human Genome Research, Huazhong University of Science and Technology,

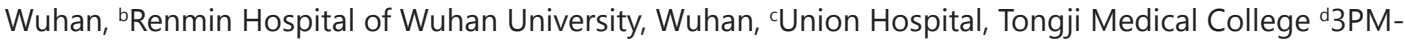
Wuhan, Wuhan, China

\section{Key Words}

Speckle-tracking based strain imaging $\bullet$ Diabetic cardiomyopathy $•$ Cardiac deformation

\begin{abstract}
Background/Aims: Diabetes mellitus (DM) has been demonstrated to have a strong association with heart failure. Conventional echocardiographic analysis cannot sensitively monitor cardiac dysfunction in type I diabetic Akita hearts, but the phenotype of heart failure is observed in molecular levels during the early stages. Methods: Male Akita (Ins2 $\left.2^{\mathrm{WT} / C 96 \mathrm{~V}}\right)$ mice were monitored with echocardiographic imaging at various ages, and then with conventional echocardiographic analysis and speckle-tracking based strain analyses. Results: With speckletracking based strain analyses, diabetic Akita mice showed changes in average global radial strain at the age of 12 weeks, as well as decreased longitudinal strain. These changes occurred in the early stage and remained throughout the progression of diabetic cardiomyopathy in Akita mice. Speckle-tracking showed that the detailed and precise changes of cardiac deformation in the progression of diabetic cardiomyopathy in the genetic type I diabetic Akita mice were uncoupled. Conclusions: We monitored early-stage changes in the heart of diabetic Akita mice. We utilize this technique to elucidate the underlying mechanism for heart failure in Akita genetic type I diabetic mice. It will further advance the assessment of cardiac abnormalities, as well as the discovery of new drug treatments using Akita genetic type I diabetic mice.
\end{abstract}




\section{Cellular Physiology Cell Physiol Biochem 2018:45:1541-1550

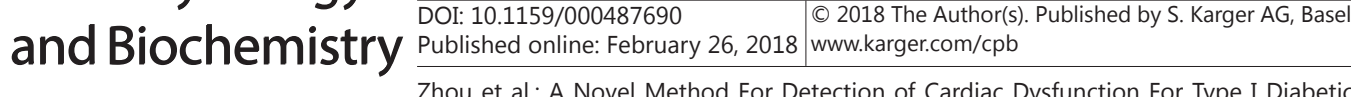 \\ Mice}

\section{Introduction}

In developed countries, diabetes mellitus (DM) was diagnosed one in ten individuals in 2013. Epidemiologic studies have long demonstrated a strong association with heart failure and diabetes [1]. The Framingham study showed that diabetes results in a roughly 2 -fold higher risk of heart failure in men and a 5-fold increase in risk in women [2]. Indeed, in the Candesartan in Heart failure-Assessment of Reduction in Mortality and morbidity (CHARM) program, diabetes was an independent predictor of morbidity and mortality in patients with heart failure, and a worse prognosis after myocardial infarction (MI) [3, 4].

Meanwhile, animal models of diabetes have led to strong evidences supported the association between diabetes and heart failure in both type I diabetes and type III diabetes $[5,6]$. To clarify the detailed mechanism, extensive experiments involved a genetic model of insulin-dependent type IIII diabetes using the Ins $2^{\text {WT/C96Y }}$ Akita mouse [7]. However, several studies uncovered that there were no significant difference in left ventricle ejection fraction (LVEF), left ventricle fraction shortening(LVFS), and stroke volume between Akita and wildtype mice at 3-months old and 6-months of age, reflecting that the systolic function of Akita mice is not significantly different from that of littermate wild-type mice [8]. Additionally, LVEDD, LVESD, and IVS were unchanged in Akita mice, showing that the structure of left ventricle is not affected by hyperglycemia in Akita mice [8]. Furthermore, no difference in E/A ratio between Akita mice and littermate wild-type mice has been reported [8]. Interestingly, other diastolic function parameters were different among Akita mice and wide mice matched for age. Although this reveals information on diastolic dysfunction in Akita mice, it is still very difficult to monitor the progression of early-stage cardiac dysfunction in Akita mice. Thus far, there is no effective and productive method to depict the process of diastolic dysfunction in type I diabetes- Akita mice.

Recently, speckle tracking echocardiography emerged to overcome the limitations of conventional ultrasound methods $[9,10]$. It is used for the estimation of the radial and circumferential strain and strain rate (SR) of myocardium in the parasternal short-axis view in mouse models, as well as longitude strain and SR in the long-axis view, reflecting the biomechanical properties in three dimensions $[11,12]$. It has been previously reported that strain is much more sensitive and can be used to detect the cardiac mechanic change in ISOinduced mice hypertrophy at early stage [13].

In this study, we present a novel echocardiographic imaging methodology that utilizes speckle-tracking-based strain analysis, which allows for non-invasive and highly sensitive cardiac phenotyping in diabetic cardiomyopathy. We show that speckle-tracking based strain analysis can efficiently monitor subtle changes in abnormal cardiac deformation in diabetic cardiomyopathy. Additionally, speckle-tracking based strain analysis could be utilized in the evaluation of emerging cardiac therapies in diabetic cardiomyopathy.

\section{Materials and Methods}

\section{Experimental animals and mouse model generation}

In vivo experiments were approved by ICUCA and performed in accordance with the Guide for the care and use of laboratory animals. Female C57BL/6 female mice (Ins2 $2^{\text {WT/WT }}$ ) were mated with male Akita (Ins2 $2^{\text {WT/ }}$ ${ }^{\mathrm{C} 96 \mathrm{Y}}$ ) at an animal facility. Only male C57BL/6 wild-type and Akita mice were used in the experiments. During the experiment, the mice were housed at controlled temperature $\left(22 \pm 1{ }^{\circ} \mathrm{C}\right)$ and humidity $(55 \%)$ with a 12:12 hours dark-light cycle; they also had unrestricted access to food and water. When required, animals were anesthetized with isoflurane (Abbott Laboratories, Illinois, USA). Before sacrificing, the mice were deeply anesthetized with $60 \mathrm{mg} / \mathrm{kg}$ ketamine plus $4 \mathrm{mg} / \mathrm{kg}$ xylazine.

Conventional echocardiographic imaging and analysis

After each mouse was anesthetized in the chamber with $4 \%$ isoflurane and $10 \%$ oxygen, the mouse was transferred and placed onto a heated platform, and maintained at $0.8 \%$ isofurane for the duration of 


\section{Cellular Physiology Cell Physiol Biochem 2018;45:1541-1550

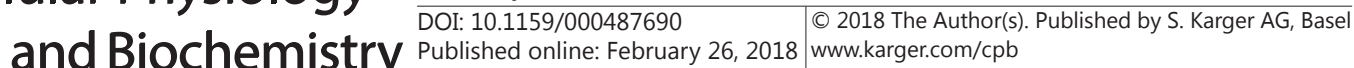 Zhou et al.: A Novel Method For Detection of Cardiac Dysfunction For Type I Diabetic \\ Mice}

the experiment [14]. Electrode gel was applied for electrocardiogram, and to determine the respiration rate, and other physiological signals. The chest was shaved, and mice were allowed to recover and be stable for at least 15 min. Ultrasound images were obtained with a MD550D transducer (22-55 MHz) on the Vevo 3100 Imaging System (Visual Sonics, Toronto, Canada). After placing the transducer to the left of the sternum, transducer position was adjusted to allow us to obtain long-axis two-dimensional B-mode images including the aortic outflow tract, the apex of the heart, and LV along its longest axis. Once all long-axis B/M-mode images were attained, the transducer was rotated 90 degrees to acquire B-mode images at the mid-papillary muscle level in the parasternal left ventricle short-axis view. Additionally, M-mode images were taken by placing a gate through the center of the B-mode images to obtain M-mode recordings of internal parameters of the myocardium. All images were acquired using the highest possible frame rate (233-401 frames/s) depending on the imaging axis to get the best possible image resolution for speckle-tracking based strain analyses. As much as possible to avoid the interference of anesthetic condition and temperature during these examination, the heart rates of mice were maintained over 400 beats/min and kept warmed.

To monitoring the diastolic function for these mice, we performed pulsed-wave Doppler to obtain the transmittal flow velocity waveforms using a four-chamber view. Because of rapid physiological heart rate of the mouse, resulting in partial or complete fusion of $\mathrm{E}$ and A waves, we acquired images of mitral flow velocity at low heart rate (less than 400 beats/ min). Color Doppler imaging were used to confirm the position of bicuspid valve and the direction of transmittal flow, therefore, it is very critical to acquire the precise transmittal flow measurements of ventricular filling velocity.

Primarily, conventional echocardiographic parameters were analyzed and calculated from M-mode parasternal short-axis B-mode images at the mid-papillary muscle level, including anterior and posterior wall thickness at both systole and diastole, fractional shortening (FS), ejection fraction (EF), cardiac output (CO), and end-diastolic and end-systolic diameters and volumes. All M-mode image measurements were performed in end-diastole and end-systole according to the American Society of Echocardiography or calculated for three consecutive cardiac cycles and then averaged.

The function of LV relaxation was evaluated according to transmittal flow velocity waveforms with pulsed-wave Doppler, mainly reflecting as the E/A ratio, deceleration time (DT), and isovolumetric relaxation time (IVRT) over three cardiac cycles. A decrease in the E/A ratio is a strong indicator of diastolic dysfunction. The MPI, Tei, is another noninvasive indicator of systolic and diastolic function (MPI = (IVRT+IVCT)/ET), according to the images from transmittal flow velocity waveforms. An increase in the MPI is a reliable predictor of diastolic dysfunction.

\section{Speckle tracking based strain imaging and analysis}

Strain analysis was performed offline using a speckle tracking algorithm with the Visual Sonics VevoStrain software (Visual Sonics, Toronto, Canada). Based on the B-model video loops at the mid-papillary muscle level in short-axis view, strain and strain rate was quantified in the radial and circumferential dimensions. Additionally, strain and strain rate in longitudinal dimension were calculated using B-mode videos acquired in the parasternal long-axis view. After strain analyses, values were positive or negative because of the assessed measurement. A negative value indicates fiber shortening, occurring in the circumferential or longitudinal dimensions, whereas a positive value for strain shows fiber lengthening or thickening in the radial dimension.

In brief, high-frequency B-mode loops were chosen from echocardiographic video in long-axis or short-axis view, making it easy to automatically track the borders of endocardium and epicardium and then check if the automatic depict was correct and if it was sufficient for three cardiac cycles frame by frame. After tracking, the data was analyzed by software, and included the average global value of strain, strain rate, velocity, and displacement. In short-axis view, average peak global values were calculated from six independent anatomical segments of the left ventricle.

\section{Plasma biochemical measurements}

Fasting blood glucose was detected with blood glucose meter (ReliOn Prime, Bentonville, AR, US), and random plasma insulin concentration was measured by ELISA (Alpco Diagnostics, Salem, NH), as previously described. 


\section{Cellular Physiology Cell Physiol Biochem 2018:45:1541-1550 \begin{tabular}{l|l|l} 
and Biochemistry Published online: February 26, 2018 & $\begin{array}{l}\text { (c) } 2018 \text { The Author(s). Published by S. Karger AG, Basel } \\
\text { www.karger.com/cpb }\end{array}$ \\
\hline
\end{tabular} \\ Zhou et al:: A Novel Method For Detection of Cardiac Dysfunction For Type I Diabetic Mice}

\section{Realtime PCR assay}

Total RNA from heart tissues were extracted using TRIZOL (Life Technologies). cDNA were synthesized using MMLV reserve transcriptase (Promega, WI, USA) with random primers. Realtime PCR analysis was performed with Power SYBR Green Master Mix (Life Technologies) in a lightCycler1.5 Instrument (Roche, Mannheim, Germany). Data were analyzed by the a- $\Delta \Delta$ Ct method, comparing threshold cycles first to $\beta$-actin expression, and then $\Delta \mathrm{Ct}$ of target genes in controls [15].

The sequences of primers used for realtime RT-PCR analysis are as following: mouse Anp (sense primer, 5'-TTC TTC CTC GTC TTG GCC TTT-3' and antisense primer, 5'- GAC CTC ATC TTC TAC CGG CAT CT-3'), mouse Bnp (sense primer, 5'-CAC CGC TGG GAG GTC ACT-3' and antisense primer, 5'-GTG AGG CCT TGG TCC TTC AAG GTC ACT-3'), mouse $\beta M h c$ (sense primer, 5'- ATG TGC CGG ACC TTG GAA - $3^{\prime}$ and antisense primer, 5'CCT CGG GTT AGC TGA GAG ATC A -3'), mouse $\beta$-actin (sense primer, 5'-CGT CCA CCC GCG AGC ACA G-3' and antisense primer, 5'- CGA CGA CCA GCG CAG CGA TAT-3').

\section{Statistical analysis}

All data were reported as mean SD. Comparisons between different groups at one time point were analyzed using one-way ANOVA with Tukey-post-test or Bonferroni-post-test (SPSS, version 19). Twoway ANOVA were used to compare between two groups at different time points.A $P<0.05$ was considered statistically significant.

\section{Results}

Body weight, heart weight, blood glucose and heart rate measurements

Body weight (BW), heart weight (HW), blood glucose, and insulin levels of type I diabetic Akita mice and wild-type male mice ( $\mathrm{n}=10$ for each group) are described in Tab 1. Body weight shows significant differences from that 8-weeks of age. Heart weights in Akita mice were less than in wildtype control at 8 weeks of age. However, the ratios of heart weight to body weight and heart weight to tibia length were not different at 16 weeks of age, indicating that the reduction in heart weight paralleled the general catabolic state of Akita mice. Surprising, cardiac mRNA levels of Anp, $B n p$, and $\beta$-myosin heavy chain ( $\beta \mathrm{MHC}$ ) were sharply increased in cardiac tissues from Akita mice compared to those in wild-type mice at 12 week of age (Fig. 1). Fasting blood glucose levels were sharply increased in Akita mice at 8 weeks of age (Wild-type, $174.8 \pm 21.6 \mathrm{mg} / \mathrm{dl}$; Akita, $368.7 \pm 63.2 \mathrm{mg} / \mathrm{dl}, P<0.01$ ) (Table 1). Additionally, the high levels of fasting blood glucose were maintained in Akita mice up to 16 weeks of age. This founding was consistent with what previously reported, and further confirm genetic type I diabetic Akita mouse is not affected with diabetic cardiomyopathy in the early stage.

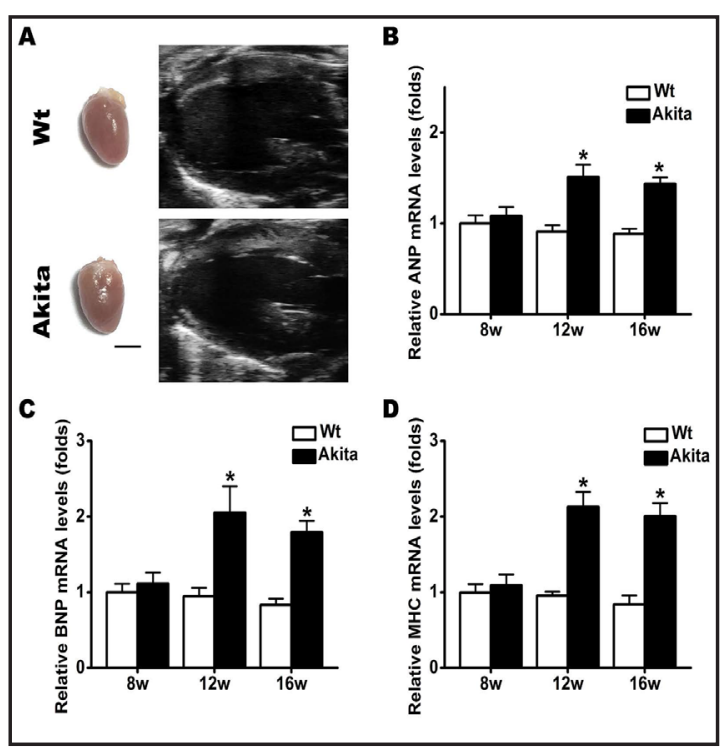

Fig. 1. Akita heart exhibit heart failure in molecular levels. A. Representative hearts from wild-type and Akita mice. Akita mouse hearts are smaller than that wild-type mouse. The right panel shows representative B-mode echocardiographic images in parasternal long-axis view from wild-type and Akita mouse. B. Anp mRNA levels in heart tissues from wild-type and Akita mice at different ages. C. Bnp mRNA levels in heart tissues from Akita mice were increased compared with those from wild-type miceat 12 weeks of age. D. Increases in $\beta M H C$ mRNA levels in heart tissues from Akita mice compared to that wild-type mice at 12 weeks of age was noted. Values are means \pm SD. $*, P<0.05$. 


\section{Cellular Physiology Cell Physiol Biochem 2018:45:1541-1550 \begin{tabular}{ll|l} 
and BiOChemistry & $\begin{array}{l}\text { DOI: 10.1159/000487690 } \\
\text { Published online: February 26, } 2018\end{array}$ & $\begin{array}{l}\text { C } 2018 \text { The Author(s). Published by S. Karger AG, Basel } \\
\text { www.karger.com/cpb }\end{array}$ \\
\hline
\end{tabular} \\ Zhou et al:: A Novel Method For Detection of Cardiac Dysfunction For Type I Diabetic Mice}

Conventional echocardiographic imaging and analysis for Akita mouse

To analyze the cardiac function at the early stage in the development of heart failure in Akita mice, mice were imaged at 8 weeks, 12 weeks and 16 weeks of age with ultrasound scanning. Conventional echocardiographic indexes of left ventricle structure and systolic function in wild-type and Akita mice at three different time points were showed in Table 2 and Fig 2. For cardiac structure, M-mode images in shortaxis view showed that the anterior and posterior wall thicknesses at the end of diastolic stage and end of systolic stage in Akita mice at 8 weeks of age were the same as those in age-matched mice (Fig. 2A and 2B). There were also no significantly different in the diameters of left ventricle between Akita and wide type mice (Table 2). Furthermore, M-mode images also showed that the systolic function in Akita mice was the same as that in wild-type mice, because parameters of cardiac systolic function, such as LVEF and LVFS, are not comparable between Akita and wild-type mice at 8 weeks of age (Fig. 2C and 2D). Meanwhile, at this time point, there was no obvious difference in cardiac output (CO) in Akita mice compared with that in wide-type mice (Fig. 2E and 2F). We further examined the cardiac structure and the systolic function in Akita and wild-

Fig. 2. Echocardiographic assessment of systolic function in 8 weeks, 12 weeks, and 16 weeks old mice. A. Representative B-mode echocardiographic images in parasternal short-axis view from wild-type and Akita mouse. B. Representative M-mode echocardiographic images in parasternal short-axis view from wild-type and Akita mouse. The curves depict the left ventricle anterior wall and the left ventricle posterior wall (LVPW). C-F. Mean parameter values for systolic function by echocardiographic assessment. No changes between wild-type and Akita mice were noted in left ventricle ejection fraction (LVEF), left ventricle fraction shorten (LVFS), ejection time (ET), or cardiac output (CO), at different ages old. Values are shown as means \pm SD. ${ }^{*}, P<0.05$.
Table 1. Characteristics of wild type and Akita mice. Values are shown as means \pm SD; n, number of mice. HW, heart weight; BW, body weight; TL, tibia length

\begin{tabular}{|c|c|c|c|}
\hline Variables & WT & Akita & P-value \\
\hline \multicolumn{4}{|l|}{8 weeks } \\
\hline $\mathrm{n}$ & 10 & 10 & \\
\hline Body weight (g) & $22.81 \pm 0.71$ & $20.72 \pm 0.49$ & $<0.01$ \\
\hline Heart Weight (g) & $104.1 \pm 3.54$ & $95.4 \pm 4.27$ & $<0.01$ \\
\hline $\mathrm{HW} / \mathrm{BW}$ & $4.57 \pm 0.20$ & $4.60 \pm 0.13$ & 0.64 \\
\hline $\mathrm{HW} / \mathrm{TL}$ & $16.01 \pm 0.10$ & $14.28 \pm 0.19$ & 0.1 \\
\hline Fasting blood glucose (mmol/L) & $174.8 \pm 21.6$ & $368.7 \pm 63.2$ & $<0.01$ \\
\hline Insulin (pM) & $78.05 \pm 2.24$ & $41.42 \pm 2.91$ & $<0.01$ \\
\hline \multicolumn{4}{|l|}{12 weeks } \\
\hline $\mathrm{n}$ & 10 & 10 & \\
\hline Body weight $(\mathrm{g})$ & $25.77 \pm 0.46$ & $22.57 \pm 0.84$ & $<0.01$ \\
\hline Heart Weight (g) & $116.1 \pm 3.57$ & $103.6 \pm 3.13$ & $<0.01$ \\
\hline $\mathrm{HW} / \mathrm{BW}$ & $4.51 \pm 0.14$ & $4.59 \pm 0.13$ & 0.16 \\
\hline $\mathrm{HW} / \mathrm{TL}$ & $17.44 \pm 0.40$ & $15.68 \pm 0.43$ & 0.3 \\
\hline Fasting blood glucose (mmol/L) & $176.4 \pm 21.0$ & $506.9 \pm 74.3$ & $<0.01$ \\
\hline Insulin (pM) & $81.09 \pm 2.78$ & $39.66 \pm 2.25$ & $<0.01$ \\
\hline \multicolumn{4}{|l|}{16 weeks } \\
\hline $\mathrm{n}$ & 10 & 10 & \\
\hline Body weight (g) & $26.63 \pm 0.82$ & $23.9 \pm 0.44$ & $<0.01$ \\
\hline Heart Weight (g) & $121.9 \pm 4.28$ & $108.8 \pm 3.26$ & $<0.01$ \\
\hline $\mathrm{HW} / \mathrm{BW}$ & $4.58 \pm 0.12$ & $4.55 \pm 0.13$ & 0.66 \\
\hline $\mathrm{HW} / \mathrm{TL}$ & $18.24 \pm 0.29$ & $16.46 \pm 0.45$ & 0.34 \\
\hline Fasting blood glucose ( $\mathrm{mmol} / \mathrm{L})$ & $173.1 \pm 20.6$ & $513.7 \pm 39.0$ & $<0.01$ \\
\hline Insulin (pM) & $83.00 \pm 2.70$ & $38.46 \pm 2.76$ & $<0.01$ \\
\hline
\end{tabular}

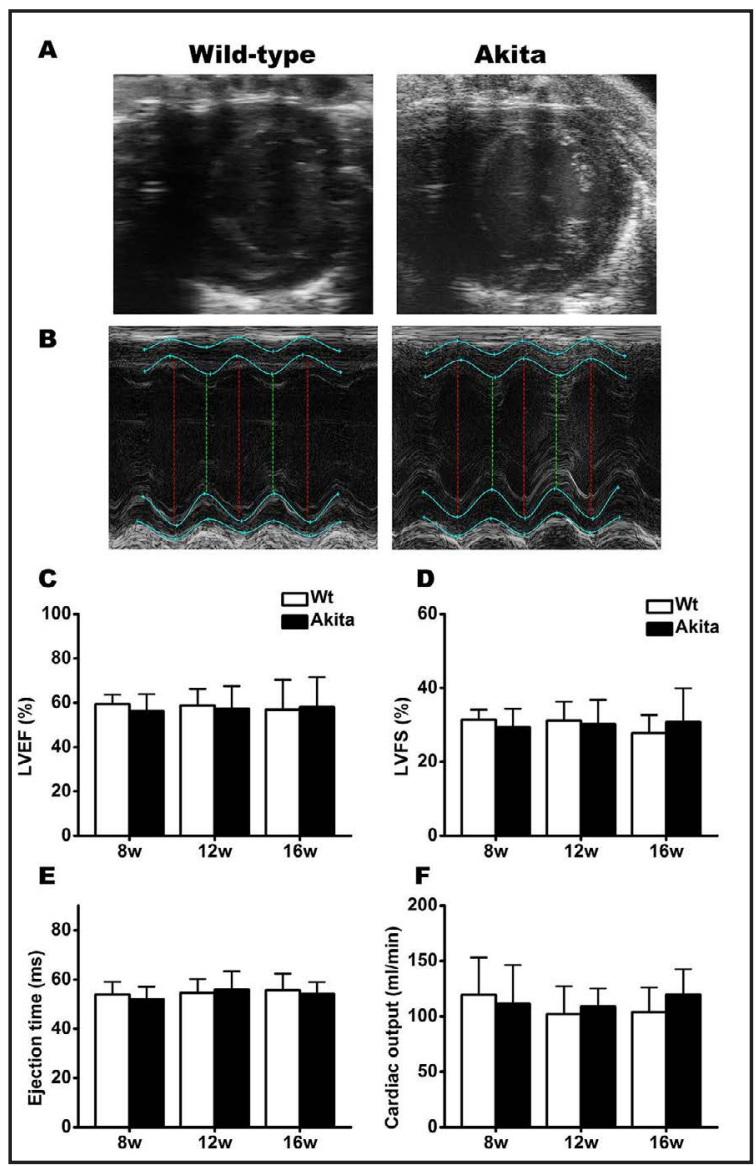




\section{Cellular Physiology Cell Physiol Biochem 2018;45:1541-1550 and Biochemistry Published online: February 26, $2018 \begin{aligned} & \text { DOI: } 2018 \text { The Author(s). Published by S. Karger AG, Basel } \\ & \text { www.karger.com/cpb }\end{aligned}$ \\ Zhou et al:: A Novel Method For Detection of Cardiac Dysfunction For Type I Diabetic Mice}

type mice at 16 week of age, with no significant difference in systolic function compared to that in wide-type mice. We evaluated diastolic function from Doppler Images from Akita and wild-type mice in 4-chamber view axis. Fig. 3A showed representative images of pulse-wave Doppler at the mitral. In contrast to wild-type mice, Akita mice showed no obvious changes in E-wave or A-wave at 8 weeks of age (Fig. 3B and 3C). Additionally, no change was identified in mice at 16 week of age. Another important parameter for diastolic function is E/A ratio. The $\mathrm{E} / \mathrm{A}$ ratios of Akita mice at 8 weeks and 16 weeks of age were not obviously different with age-matched wild-type mice (Fig. 3D). IVRT, a measurement of the time from the closing of the aortic valve to the onset of mitral flow, was not different between diabetic and wildtype mice at the age of 8 weeks or 12 weeks (Fig. 3E). Interestingly, at 16 weeks of age, IVRT in Akita mice was significantly increased compared with that in aged-matched mildtype, showing relaxation was impaired (Wildtype, $12.21 \pm 2.01 \mathrm{~ms}$; Akita, $14.44 \pm 1.39$ ms, $P=0.035$ ) (Fig. 3E). The MPI was also not increased markedly in diabetic Akita mice. These results showed that Akita mice at 16 weeks of age exhibit partial of diastolic dysfunction than age-matched wild-type mice, mainly suggesting improved cardiac relaxation (Fig. 3F). However, these results collectively indicated that these parameters of diastolic function are not sensitive and efficient for monitor progression in the early stage of diabetic cardiomyopathy in genetic type I diabetic Akita mice.
Table 2. Cardiac structure in wild-type and Akita mice at different time points. Values are means \pm SD. HR, heart rate; IVS, interventricular septum (left ventricle anterior wall, LVAW); LVID, left ventricular interior diameter; LVPW, left ventricle posterior wall; d, diastole: s, systole

\begin{tabular}{lccc}
\hline Variables & WT & Akita & P-value \\
\hline 8 weeks & & & \\
HR(beats /min) & $412 \pm 49$ & $453 \pm 70$ & 0.23 \\
IVS;d (mm) & $0.60 \pm 0.10$ & $0.56 \pm 0.16$ & 0.61 \\
IVS;s (mm) & $0.96 \pm 0.19$ & $0.95 \pm 0.26$ & 0.92 \\
LVID;d (mm) & $4.55 \pm 0.53$ & $4.34 \pm 0.33$ & 0.45 \\
LVID;s (mm) & $3.14 \pm 0.49$ & $3.06 \pm 0.42$ & 0.77 \\
LVPW;d (mm) & $0.62 \pm 0.11$ & $0.59 \pm 0.17$ & 0.69 \\
LVPW;s (mm) & $1.00 \pm 0.15$ & $0.99 \pm 0.32$ & 0.99 \\
12 weeks & & & \\
HR(beats/min) & $430 \pm 29$ & $459 \pm 37$ & 0.13 \\
IVS;d (mm) & $0.61 \pm 0.10$ & $0.60 \pm 0.13$ & 0.88 \\
IVS;s (mm) & $1.07 \pm 0.18$ & $0.89 \pm 0.24$ & 0.17 \\
LVID;d (mm) & $4.36 \pm 0.33$ & $4.34 \pm 0.60$ & 0.94 \\
LVID;s (mm) & $3.01 \pm 0.39$ & $3.06 \pm 0.68$ & 0.88 \\
LVPW;d (mm) & $0.59 \pm 0.19$ & $0.53 \pm 0.18$ & 0.4 \\
LVPW;s (mm) & $0.97 \pm 0.28$ & $0.88 \pm 0.24$ & 0.58 \\
16 weeks & & & \\
HR(beats /min) & $491 \pm 87$ & $433 \pm 42$ & 0.11 \\
IVS;d (mm) & $0.67 \pm 0.16$ & $0.63 \pm 0.11$ & 0.42 \\
IVS;s (mm) & $0.98 \pm 0.25$ & $0.95 \pm 0.23$ & 0.41 \\
LVID;d (mm) & $4.31 \pm 0.37$ & $3.85 \pm 0.62$ & 0.15 \\
LVID;s (mm) & $3.19 \pm 0.48$ & $2.71 \pm 0.63$ & 0.16 \\
LVPW;d (mm) & $0.56 \pm 0.12$ & $0.46 \pm 0.18$ & 0.27 \\
LVPW;s (mm) & $0.76 \pm 0.20$ & $0.53 \pm 0.36$ & 0.18 \\
\hline & & &
\end{tabular}

Measurement of myocardial mechanic properties at the early stage in the progression of diabetic cardiomyopathy in Akita mice

With speckle tracking based strain analysis, left ventricular functional can be examined in the radial and circumferential dimensions the short axis, partly reflecting the deformation of myocardial fiber. To completely monitor the deformation of the whole left ventricle, we traced the endocardium and epicardium frame-to-frame during the cine loop, providing assessment of the deformation of the different parts of heart tissue. Regarding the endocardium, no obvious differences were observed in short-axis average radial velocity, displacement, strain, or strain rate in Akita mice at 8 weeks of age compared with those aged-matched wild -type mice. An increase in strain was noted in Akita mice at week 12 when compared to wild-type mice. At 16 weeks of age, an increase in average radial strain was apparent in Akita mice (Table 3). No differences in circumferential strain, strain rate, velocity, or displacement in the endocardium were observed between Akita mice and wildtype mice at different ages of 8,12 and 16 weeks.

Subsequently, strain analysis was undergone in the epicardium in short axis. Additionally, an increase in average radial strain was noted in Akita mice at 12 weeks of age which had 
Fig. 3. Pulse-wave Doppler imaging for diastolic function in Akita mice compared with littermate wildtype mice. A. Representative transmitral pulse-wave Doppler flow profiles in four-chamber axis in wildtype mice and Akita mice when they were 12 weeks of age. B-D. Echocardiographic analyses showed no changes between wild-type and Akita mice in E-wave velocity, A-wave velocity, or E/A ra-

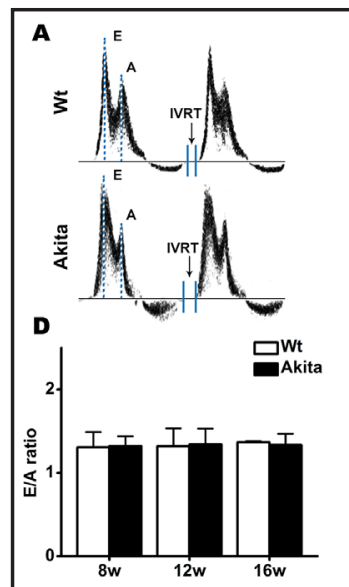

C

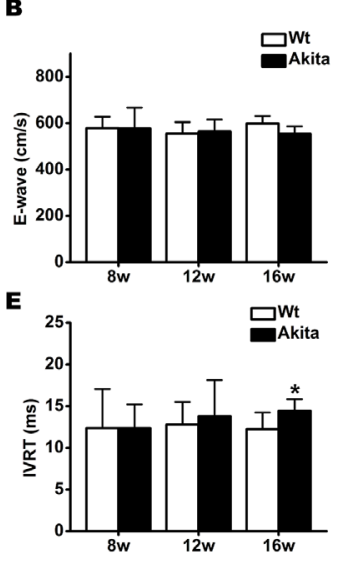

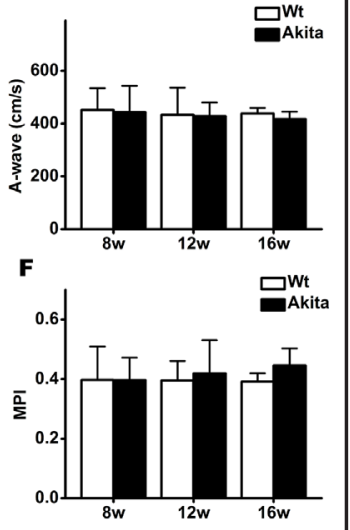

tio. E. Prolongation of the isovolumic relaxation time (IVRT) was noted in Akita mice at 16 weeks of age. F. The myocardial performance index (MPI) of mice are shown. Values are means \pm SD. *, $P<0.05$.

Table 3. Strain echocardiographic analysis for endocardium in Akita and littermate wild-type mice. Values are shown as means \pm SD

\begin{tabular}{|c|c|c|c|c|c|c|c|c|c|}
\hline & \multicolumn{3}{|c|}{8 weeks } & \multicolumn{3}{|c|}{12 weeks } & \multicolumn{3}{|c|}{16 weeks } \\
\hline & Wt & Akita & P-value & Wt & Akita & P-value & Wt & Akita & P-value \\
\hline \multicolumn{10}{|l|}{ Radial } \\
\hline Velocity $(\mathrm{cm} / \mathrm{s})$ & $1.19 \pm 0.15$ & $1.45 \pm 0.71$ & 0.39 & $1.21 \pm 0.25$ & $1.15 \pm 0.11$ & 0.56 & $1.47 \pm 0.69$ & $1.24 \pm 0.14$ & 0.42 \\
\hline Displacement (mm) & $0.48 \pm 0.5$ & $0.44 \pm 0.05$ & 0.14 & $0.47 \pm 0.08$ & $0.43 \pm 0.06$ & 0.29 & $0.46 \pm 0.05$ & $0.44 \pm 0.04$ & 0.53 \\
\hline Strain (\%) & $39.93 \pm 4.73$ & $40.31 \pm 5.86$ & 0.89 & $40.93 \pm 5.19$ & $35.29 \pm 5.37$ & 0.04 & $40.09 \pm 8.15$ & $28.81 \pm 5.69$ & 0.01 \\
\hline Strain rate $(1 / \mathrm{s})$ & $8.96 \pm 2.61$ & $12.17 \pm 7.86$ & 0.34 & $8.76 \pm 1.97$ & $7.90 \pm 0.87$ & 0.26 & $11.72 \pm 7.97$ & $7.80 \pm 1.78$ & 0.25 \\
\hline \multicolumn{10}{|l|}{ Circumferential } \\
\hline Velocity (deg/s) & $135.20 \pm 46.94$ & $243.29 \pm 189.93$ & 0.37 & $123.30 \pm 41.32$ & $193.39 \pm 97.17$ & 0.09 & $240.35 \pm 278.53$ & $165.54 \pm 80.79$ & 0.52 \\
\hline Displacement (deg) & $2.51 \pm 1.89$ & $2.92 \pm 2.42$ & & $1.89 \pm 1.24$ & $5.49 \pm 4.88$ & 0.08 & $3.56 \pm 2.17$ & $2.28 \pm 2.02$ & 0.28 \\
\hline Strain (\%) & $-21.04 \pm 2.90$ & $-20.00 \pm 2.96$ & 0.51 & $-20.71 \pm 4.09$ & $-20.98 \pm 5.26$ & 0.91 & $-20.84 \pm 2.01$ & $-20.70 \pm 3.60$ & 0.93 \\
\hline Strain rate $(1 / \mathrm{s})$ & $-6.06 \pm 1.23$ & $-8.13 \pm 4.46$ & 0.28 & $-6.43 \pm 1.60$ & -6.701 .59 & 0.73 & $-8.01 \pm 4.38$ & $-7.66 \pm 2.87$ & 0.86 \\
\hline \multicolumn{10}{|l|}{ Longitudial } \\
\hline Velocity $(\mathrm{cm} / \mathrm{s})$ & $0.63 \pm 0.20$ & $0.77 \pm 0.38$ & 0.4 & $0.77 \pm 0.28$ & 0.770 .22 & 0.99 & $0.67 \pm 0.25$ & $0.95 \pm 0.15$ & 0.3 \\
\hline Displacement (mm) & $0.15 \pm 0.08$ & $0.29 \pm 0.14$ & 0.04 & $0.24 \pm 0.13$ & 0.260 .08 & 0.66 & $0.22 \pm 0.08$ & $0.30 \pm 0.08$ & 0.06 \\
\hline Strain (\%) & $-11.40 \pm 3.81$ & $-11.84 \pm 5.57$ & 0.96 & $-12.42 \pm 2.53$ & $-12.21 \pm 4.09$ & 0.9 & $-12.59 \pm 3.32$ & $-16.30 \pm 2.51$ & 0.04 \\
\hline Strain rate $(1 / \mathrm{s})$ & $-4.03 \pm 2.32$ & $-3.32 \pm 1.23$ & 0.47 & $-3.98 \pm 1.08$ & $-3.74 \pm 0.87$ & 0.63 & $-4.01 \pm 0.96$ & $-5.42 \pm 1.77$ & 0.1 \\
\hline
\end{tabular}

Table 4. Strain echocardiographic analysis for epicardium in Akita and littermate wild-type mice. Values are shown as means $\pm \mathrm{SD}$

\begin{tabular}{|c|c|c|c|c|c|c|c|c|c|}
\hline & \multicolumn{3}{|c|}{8 weeks } & \multicolumn{3}{|c|}{12 weeks } & \multicolumn{3}{|c|}{16 weeks } \\
\hline & Wt & Akita & P-value & $\mathrm{Wt}$ & Akita & $\mathrm{P}$-value & Wt & Akita & P-value \\
\hline \multicolumn{10}{|l|}{ Radial } \\
\hline Velocity $(\mathrm{cm} / \mathrm{s})$ & $0.64 \pm 0.16$ & $0.75 \pm 0.27$ & 0.39 & $0.70 \pm 0.16$ & $0.66 \pm 0.12$ & 0.57 & $0.80 \pm 0.26$ & $0.78 \pm 0.23$ & 87 \\
\hline Displacement (mm) & $0.23 \pm 0.06$ & $0.22 \pm 0.05$ & 0.64 & $0.25 \pm 0.06$ & $0.23 \pm .03$ & 0.47 & $0.23 \pm 0.06$ & $0.28 \pm 0.09$ & 0.25 \\
\hline Strain (\%) & $39.93 \pm 4.73$ & $40.31 \pm 5.86$ & 0.89 & $40.93 \pm 5.19$ & $35.29 \pm 5.37$ & 0.04 & $40.09 \pm 8.15$ & $29.91 \pm 7.33$ & 0.03 \\
\hline Strain rate $(1 / \mathrm{s})$ & $8.96 \pm 2.61$ & $12.17 \pm 7.86$ & 0.34 & $8.76 \pm 1.97$ & $7.90 \pm 0.87$ & 0.26 & $11.72 \pm 7.97$ & $8.03 \pm 1.76$ & 0.27 \\
\hline \multicolumn{10}{|l|}{ Circumferential } \\
\hline Velocity (deg/s) & $58.69 \pm 27.24$ & $107.61 \pm 113.85$ & 0.31 & $57.55 \pm 16.09$ & $94.98 \pm 42.59$ & 0.04 & $104.46 \pm 113.01$ & $89.99 \pm 66.79$ & 0.78 \\
\hline Displacement (deg) & $1.10 \pm 0.47$ & $1.63 \pm 1.17$ & 0.39 & $1.10 \pm 0.61$ & $2.46 \pm 1.86$ & 0.08 & $1.96 \pm 1.37$ & $1.39 \pm 1.85$ & 0.52 \\
\hline Strain (\%) & $-8.00 \pm 2.47$ & $-7.77 \pm 1.87$ & 0.84 & $-9.12 \pm 2.41$ & $-8.90 \pm 1.03$ & 0.8 & $-8.07 \pm 1.43$ & $-11.33 \pm 5.53$ & 0.18 \\
\hline Strain rate $(1 / \mathrm{s})$ & $-2.46 \pm 0.75$ & $-3.31 \pm 1.87$ & 0.29 & $-2.77 \pm 0.65$ & $-2.85 \pm 0.48$ & 0.79 & $-3.33 \pm 1.84$ & $-4.06 \pm 2.91$ & 0.59 \\
\hline \multicolumn{10}{|l|}{ Longitudial } \\
\hline Velocity $(\mathrm{cm} / \mathrm{s})$ & $0.49 \pm 0.19$ & $0.57 \pm 0.19$ & 0.44 & $0.60 \pm 0.17$ & $0.53 \pm 0.17$ & 0.37 & $0.48 \pm 0.19$ & $0.58 \pm 0.15$ & 0.29 \\
\hline Displacement (mm) & $0.12 \pm 0.05$ & $0.19 \pm 0.07$ & 0.06 & $0.17 \pm 0.06$ & $0.19 \pm 0.07$ & 0.51 & $0.14 \pm 0.06$ & $0.20 \pm 0.05$ & 0.05 \\
\hline Strain (\%) & $-6.79 \pm 2.72$ & $-5.68 \pm 2.79$ & 0.45 & $-6.83 \pm 3.33$ & $-6.38 \pm 3.69$ & 0.79 & $-5.83 \pm 2.28$ & $-9.54 \pm 2.28$ & 0.01 \\
\hline Strain rate $(1 / \mathrm{s})$ & $-2.25 \pm 1.28$ & $-1.81 \pm 0.76$ & 0.42 & $-2.37 \pm 0.97$ & $-1.98 \pm 1.05$ & 0.44 & $-1.89 \pm 0.86$ & $-3.03 \pm 0.68$ & 0.02 \\
\hline
\end{tabular}




\section{Cellular Physiology Cell Physiol Biochem 2018;45:1541-1550 \\ \begin{tabular}{ll|l} 
and Biochemistry & $\begin{array}{l}\text { DOI: } 10.1159 / 000487690 \\
\text { Published online: February 26, } 2018\end{array}$ & $\begin{array}{l}\text { c } 2018 \text { The Author(s). Published by S. Karger AG, Basel } \\
\text { www.karger.com/cpb }\end{array}$ \\
\hline Whou
\end{tabular} \\ Mice}

increased more at 16 weeks of age. However there was no changes in strain rate, velocity, or displacement (Table 4).

Moreover, with speckle tracking based strain analysis, cardiac deformations in radial and longitudinal dimensions in long -axis can be monitored. After tracing the endocardium and epicardium in B-mode video, the deformations were assessed in different parts of hearts. No changes were noted in mean longitudinal strain, strain rate, velocity or displacement in both of endocardium or epicardium at 8 weeks of age or 12 weeks of age (Table 3 and Table 4). At 12 weeks of age, the longitudinal strain and strain rate in epicardium were significantly decreased in Akita mice when compared with age-matched wild-type mice (Table 4). Increases in average longitudinal strain and strain rate in endocardium were also noted in Akita mice, along with significant increase of velocity (Table 3).

\section{Discussion}

To our knowledge, this study is the first to demonstrate detailed and precise changes in abnormal cardiac deformation in early-stage of diabetic cardiomyopathy in the genetic type I diabetic Akita mice. Thus, subtle changes in cardiac function can be identified using speckle-tracking-based strain analysis. Here we describe the utility of speckle-trackingbased analysis for the detection of cardiac dysfunction in diabetic cardiomyopathy in Akita mice, especially in the early stages of the disease.

Extensive evidences have demonstrated that type I diabetic patients will occur diabetic cardiomyopathy [16]. A number of studies have elucidated the progression of diabetic cardiomyopathy, with an aim to rescue diabetic patients [17]. Streptozotocin (STZ)-induced diabetes in rodent models has been assessed to investigate the complications of type I diabetes [18]. It has been reported that STZ-induced diabetic cardiomyopathy is characterized by early diastolic and vascular dysfunction, which develops into systolic dysfunction, resulting in heart failure. This is reflected by increased LV diastolic pressure measured by catheterization, and also from abnormal patterns of mitral inflow and pulmonary venous flow using Doppler echocardiography $[19,20]$. Subsequently, decreased rates of LVEF and LVFS were noted with echocardiographic analyses [21,22]. Additionally, LV catheterization has shown reduced left ventricular (LV) systolic pressure and diminished $\pm \mathrm{dP} / \mathrm{dt}$ (rate of pressure rise or fall during systole and diastole, respectively) have been demonstrated $[19,23]$. In Akita mice, an increase of IVRT in Aktia mice was noted compared to wild-type mice at 3 and 6 month of age, as previous demonstrated. However, it was reported that other indexes for diastolic function were not stable or sensitive for monitoring, such as E-wave velocity (E), A-wave velocity (A), deceleration time (DT), E-wave deceleration rate (EWDR), and early diastolic tissue Doppler velocity (E'). Difference in E/A ratio, an essential parameter for diastolic function, was not observed in Akita mice at 8 week, 12 week, and 16 weeks of age. However, there was no difference in LVEF and LVFS between wild-type and Akita mice, another mouse model for type I diabetes, suggesting that the systolic function is not affected in Akita mice at young or old stage (Fig. 2) [24]. Surprisingly, an increase in markers for heart failure was noted in Akita mice at 12 week of age in our study, indicating that there was a subtle change in heart in Akita mice even at an young age(Fig. 1).

Because previous studies indicate that current diastolic parameters were not suitable and effective for detection of cardiac function and the progression of heart failure in Akita mice, identifying e subtle changes in diastolic function are necessary to elucidate the pathogenesis of diabetic cardiomyopathy. Currently, several clinical studies have shown that the detection of subtle changes of strain measurements in early diabetic were associated with the future development of cardiovascular disease, whereas conventional measures of cardiac structure and function were unaltered. Subsequently, for researchers, it is possible to screen such subtle and early changes in cardiac function in small animal models to assess different cardiac therapies or discovered drugs to prevent cardiac dysfunction in cardiovascular diseases. Subtle change in myocardia 


\section{Cellular Physiology Cell Physiol Biochem 2018;45:1541-1550 \\ \begin{tabular}{ll|l} 
DOI: 10.1159/000487690 & $\begin{array}{l}\text { O } 2018 \text { The Author(s). Published by S. Karger AG, Basel } \\
\text { www.karger.com/cpb }\end{array}$ \\
\hline
\end{tabular} \\ Zhou et al.: A Novel Method For Detection of Cardiac Dysfunction For Type I Diabetic \\ Mice}

mechanic properties should appear prior to cardiac morphological structure and cardiac dysfunction. It is possible that the abnormality in myocardia fiber deformation may lead to cardiac structural abnormality, which is accompanying by cardiac dysfunction, including systolic and diastolic dysfunction. High frequency ultrasound shows promise in acquiring the loop clip, overcoming the problems of fast heart beats in the mouse, and permit the adoption of speckle-tracking-based analysis.

The current literatures indicated that speckle-tracking-based strain analysis in identifying subtle early-stages changes prior to conventional measurements provides a repeatable approach to evaluate cardiac function in a small animal model with certain cardiac pathology. Experiments on small animals are more complicated and difficult to perform because of fast heart rates, small animal sizes, lacking high-tech imaging equipment and analysis systems. Our results indicated that the progression of the type I diabetic cardiomyopathy at 12 weeks of age was associated with a decrease in radial strain and strain rate compared with those littermate wild-type mice. As age increased, impairments in longitudinal strain and strain rate were magnified in Akita mice. However, changes in strain and strain rate in wild-type mice were not obvious at 8 and 12 weeks of age. It was demonstrated previously that cardiac deformations in STZ-induced type I diabetic mice were detected at the early stage with speckle tracking based analysis. It was discovered that, in STZ-induced diabetic mice, changes in average global systolic radial strain, strain rate, displacement and velocity, along with decreased circumferential and longitudinal strain rate, were noted starting from 1-week after the onset of diabetes. These changes were remained throughout the progression of the diabetic cardiomyopathy. It is earlier to identify subtle changes in strain at 1-week from the onset of diabetes than tat 6 weeks from the onset by conventional ultrasound analyses. From these results, speckle-tracking-based-strain is considered to be a novel and sensitive approach to monitor subtle changes in the early stages of such heart diseases.

In this study, we present a novel echocardiographic imaging methodology that utilizes speckle-tracking-based-strain analysis that allows for non-invasive and highly sensitive cardiac phenotyping in diabetic cardiomyopathy. We show that speckle-tracking-based strain analysis can efficiently monitor subtle changes in abnormal cardiac deformation in diabetic cardiomyopathy. Additionally, speckle-tracking-based strain analysis could be utilized in the evaluation of emerging cardiac therapies in diabetic cardiomyopathy.

\section{Acknowledgements}

We gratefully acknowledge Yufeng Yao for his contribution to the design of some parts of the current study, and other lab members for technical support and advice.

This work was supported by grants from the National Basic Research Program of China (973 Program: 2013CB531103), the National Natural Science Foundation of China (No. 91439109 and 81270163, the Program for New Century Excellent Talents at the University of China (NCET-11-0181).

\section{Disclosure Statement}

No conflict of interests exists.

\section{References}

1 Blecker S, Paul M, Taksler G, Ogedegbe G, Katz S: Heart failure-associated hospitalizations in the United States. J AM Coll Cardiol 2013;61:1259-1267.

-2 Kannel WB, Hjortland M, Castelli WP: Role of diabetes in congestive heart failure: The Framingham study. Am J Cardiol 1974;34:29-34.

3 Bell DS: Diabetic cardiomyopathy. Diabetes Care 2003;26:2949-2951.

4 MacDonald MR, Petrie MC, Varyani F, Ostergren J, Michelson EL, Young JB, Solomon SD, Granger CB, 


\section{Cellular Physiology Cell Physiol Biochem 2018:45:1541-1550 \begin{tabular}{l|l|l|l} 
DOI: 10.1159/000487690 & O 2018 The Author(s). Published by S. Karger AG, Basel \\
www.karger.com/cpb
\end{tabular} \\ Zhou et al.: A Novel Method For Detection of Cardiac Dysfunction For Type I Diabetic Mice}

Swedberg K, Yusuf S, Pfeffer MA, McMurray JJ: Impact of diabetes on outcomes in patients with low and preserved ejection fraction heart failure: An analysis of the Candesartan in Heart failure: Assessment of Reduction in Mortality and morbidity (CHARM) programme. Eur Heart J 2008;29:1377-1385.

5 Nichols GA, Gullion CM, Koro CE, Ephross SA, Brown JB: The incidence of congestive heart failure in type 2 diabetes: An update. Diabetes Care 2004;27:1879-1884.

-6 Nasir S, Aguilar D: Congestive heart failure and diabetes mellitus: Balancing glycemic control with heart failure improvement. Am J Cardiol 2012;110:50B-57B.

-7 Ashihara T, Haraguchi R, Nakazawa K, Namba T, Ikeda T, Nakazawa Y, Ozawa T, Ito M, Horie M, Trayanova NA: The role of fibroblasts in complex fractionated electrograms during persistent/permanent atrial fibrillation: Implications for electrogram-based catheter ablation. Circ Res 2012;110:275-284.

-8 Basu R, Oudit GY, Wang X, Zhang L, Ussher JR, Lopaschuk GD, Kassiri Z: Type 1 diabetic cardiomyopathy in the Akita (Ins2WT/C96Y) mouse model is characterized by lipotoxicity and diastolic dysfunction with preserved systolic function. Am J Physiol Heart Circ Physiol 2009;297:H2096-H2108.

-9 Kleinbongard P, Bose D, Baars T, Mohlenkamp S, Konorza T, Schoner S, Elter-Schulz M, Eggebrecht H, Degen H, Haude M, Levkau B, Schulz R, Erbel R, Heusch G: Vasoconstrictor potential of coronary aspirate from patients undergoing stenting of saphenous vein aortocoronary bypass grafts and its pharmacological attenuation. Circ Res 2011;108:344-352.

10 Perry R, De Pasquale CG, Chew DP, Joseph MX: Assessment of early diastolic left ventricular function by two-dimensional echocardiographic speckle tracking. Eur J Echocardiogr 2008;9:791-795.

-11 Ram R, Mickelsen DM, Theodoropoulos C, Blaxall BC: New approaches in small animal echocardiography: Imaging the sounds of silence. Am J Physiol Heart Circ Physiol 2011;301:H1765-H1780.

12 Blessberger H, Binder T: NON-invasive imaging: Two dimensional speckle tracking echocardiography: Basic principles. Heart 2010;96:716-722.

13 An X, Wang J, Li H, Lu Z, Bai Y, Xiao H, Zhang Y, Song Y: Speckle tracking based strain analysis is sensitive for early detection of pathological cardiac hypertrophy. Plos One 2016;11:e149155.

14 Lu Q Yao Y, Hu Z, Hu C, Song Q, Ye J, Xu C, Wang AZ, Chen Q, Wang QK: Angiogenic factor AGGF1 activates autophagy with an essential role in therapeutic angiogenesis for heart disease. Plos Biol 2016;14:e1002529.

15 Lu Q, Yao Y, Yao Y, Liu S, Huang Y, Lu S, Bai Y, Zhou B, Xu Y, Li L, Wang N, Wang L, Zhang J, Cheng X, Qin G, Ma W, Xu C, Tu X, Wang Q: Angiogenic factor AGGF1 promotes therapeutic angiogenesis in a mouse limb ischemia model. Plos One 2012;7:e46998.

16 de Ferranti SD, de Boer IH, Fonseca V, Fox CS, Golden SH, Lavie CJ, Magge SN, Marx N, McGuire DK, Orchard TJ, Zinman B, Eckel RH: Type 1 diabetes mellitus and cardiovascular disease: A scientific statement from the American Heart Association and American Diabetes Association. Circulation 2014;130:1110-1130.

17 von Herrath M, Nepom GT: Animal models of human type 1 diabetes. Nat Immunol 2009;10:129-132.

18 King AJ: The use of animal models in diabetes research. Br J Pharmacol 2012;166:877-894.

19 Kajstura J, Fiordaliso F, Andreoli AM, Li B, Chimenti S, Medow MS, Limana F, Nadal-Ginard B, Leri A, Anversa P: IGF-1 overexpression inhibits the development of diabetic cardiomyopathy and angiotensin II-mediated oxidative stress. Diabetes 2001;50:1414-1424.

-20 Lacombe VA, Viatchenko-Karpinski S, Terentyev D, Sridhar A, Emani S, Bonagura JD, Feldman DS, Gyorke S, Carnes CA: Mechanisms of impaired calcium handling underlying subclinical diastolic dysfunction in diabetes. Am J Physiol Regul Integr Comp Physiol 2007;293:R1787-R1797.

-21 Nielsen LB, Bartels ED, Bollano E: Overexpression of apolipoprotein B in the heart impedes cardiac triglyceride accumulation and development of cardiac dysfunction in diabetic mice. J Biol Chem 2002;277:27014-27020.

22 Suarez J, Scott B, Dillmann WH: Conditional increase in SERCA2a protein is able to reverse contractile dysfunction and abnormal calcium flux in established diabetic cardiomyopathy. Am J Physiol Regul Integr Comp Physiol 2008;295:R1439-R1445.

23 Van Linthout S, Seeland U, Riad A, Eckhardt O, Hohl M, Dhayat N, Richter U, Fischer JW, Bohm M, Pauschinger M, Schultheiss HP, Tschope C: Reduced MMP-2 activity contributes to cardiac fibrosis in experimental diabetic cardiomyopathy. Basic Res Cardiol 2008;103:319-327.

24 Bugger H, Boudina S, Hu XX, Tuinei J, Zaha VG, Theobald HA, Yun UJ, McQueen AP, Wayment B, Litwin E, Abel ED: Type 1 diabetic akita mouse hearts are insulin sensitive but manifest structurally abnormal mitochondria that remain coupled despite increased uncoupling protein 3. Diabetes 2008;57:2924-2932. 